Portland State University

PDXScholar

3-16-2020

\title{
State Variability in the Prevalence and Healthcare Utilization of Assisted Living Residents with Dementia
}

\author{
Kali S. Thomas \\ Brown University \\ Wenhan Zhang \\ US department of Veterans Affairs \\ Portia Y. Cornell \\ Brown University \\ Lindsey Smith \\ Portland State University \\ Brian Kaskie \\ University of lowa
}

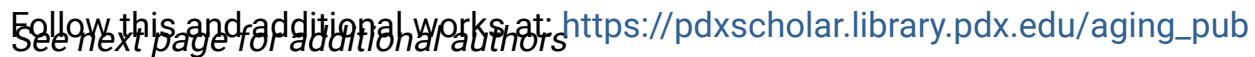

Part of the Geriatrics Commons

Let us know how access to this document benefits you.

\section{Citation Details}

Thomas, K.S., Zhang, W., Cornell, P.Y., Smith, L., Kaskie, B. and Carder, P.C. (2020), State Variability in the Prevalence and Healthcare Utilization of Assisted Living Residents with Dementia. J Am Geriatr Soc, 68: 1504-1511. doi:10.1111/jgs.16410

This Article is brought to you for free and open access. It has been accepted for inclusion in Institute on Aging Publications by an authorized administrator of PDXScholar. Please contact us if we can make this document more accessible: pdxscholar@pdx.edu. 
Authors

Kali S. Thomas, Wenhan Zhang, Portia Y. Cornell, Lindsey Smith, Brian Kaskie, and Paula C. Carder 


\title{
State Variability in the Prevalence and Healthcare Utilization of Assisted Living Residents with Dementia
}

\author{
Kali S. Thomas, PhD, * Wenhan Zhang, MPH, ${ }^{\dagger}$ Portia Y. Cornell, PhD, ${ }^{*}$ Lindsey Smith, MPP, \\ Brian Kaskie, $\mathrm{PhD},{ }^{\S}$ and Paula C. Carder, $\mathrm{PhD}^{\neq}$
}

OBJECTIVES: Almost 1 million older and disabled adults who require long-term care reside in assisted living (AL), approximately $40 \%$ of whom have a diagnosis of Alzheimer's disease and related dementias (ADRD). States vary in their regulations specific to dementia care that may influence the presence of residents with ADRD in AL and their outcomes. The objectives of this study were to describe the state variability in the prevalence of ADRD among Medicare beneficiaries residing in larger $(25+$ bed) ALs and their healthcare utilization.

DESIGN: Retrospective observational national study.

PARTICIPANTS: National cohort of 293,336 Medicare fee-for-service enrollees residing in larger $(25+$ bed $)$ ALs in 2016 and 2017 including 88,867 (30.3\%) residents with ADRD. We compared this cohort's characteristics and healthcare utilization with that of individuals with ADRD who resided in nursing homes (NHs; $\mathrm{n}=602,521$ ) and the community ( $\mathrm{n}=2,074,420$ ).

METHODS: Medicare enrollment data, claims, and the NH Minimum Data Set were used to describe differences among ADRD patients in $\mathrm{AL}, \mathrm{NHs}$, and the community. We present rates of $\mathrm{NH}$ admission and hospitalization, by state, adjusting for age, sex, race, dual eligibility, and chronic conditions.

RESULTS: The prevalence of ADRD among AL residents varied by state, ranging from $24 \%$ to $47 \%$. In 2017 , AL residents with ADRD had higher rates of $\mathrm{NH}$ admission than their community-dwelling counterparts (adjusted national average $=24 \%$, ranging from $14 \%$ to $35 \%$ among states).

From the *Brown University School of Public Health, U.S. Department of Veterans Affairs Medical Center, Providence, Rhode Island; ${ }^{\dagger}$ Center for Gerontology and Healthcare Research, Brown University School of Public Health, Providence, Rhode Island; "Institute on Aging, Portland State University, Portland, Oregon; and the ${ }^{\S}$ Department of Health Management and Policy, University of Iowa, Iowa City, Iowa.

Address correspondence to Kali S. Thomas, PhD, Box G-S121(6), 121 S. Main Street, Providence, RI 02912. E-mail: kali_thomas@brown. edu. Twitter: @kalit23

Meeting Presentations: Material from this article was presented at the 2019 Annual Meetings of the American Geriatrics Society, AcademyHealth, and Gerontological Society of America.

DOI: 10.1111 /jgs.16410
AL residents with ADRD had higher rates of hospitalization $(38 \%)$ than populations in either $\mathrm{NHs}(29 \%)$ or the community $(34 \%)$, and ranged from $29 \%$ to $45 \%$ of residents among states.

CONCLUSION: These findings have implications for states as they regulate $\mathrm{AL}$ and for healthcare professionals whose patients reside in AL. Future work is needed to understand specific elements of states' regulatory environments and local markets that may impact access and outcomes for this vulnerable population of residents with ADRD. J Am Geriatr Soc 68:1504-1511, 2020.

Keywords: assisted living; Alzheimer's disease and related dementias; long-term care

$\mathrm{O}$ ver the past 2 decades, assisted living (AL) has rapidly emerged as a preferred residence for many older and disabled adults who require long-term care. Each day in 2016, approximately $29,000 \mathrm{AL}$ residences were home to more than 800,000 people. ${ }^{1}$ Studies vary on their prevalence estimates of cognitive impairment or an Alzheimer's disease and related dementias (ADRD) diagnosis among $\mathrm{AL}$ residents, and range anywhere from $40 \%$ to $72 \%{ }^{1-7}$ Estimates derived from the National Survey of Residential Care Facilities suggest that in 2010, 7 of 10 AL residents had some form of cognitive impairment, with $19 \%$ exhibiting severe cognitive impairment. ${ }^{3}$ Persons with ADRD residing in AL represent a population with an increased risk of poor outcomes. For example, persons with ADRD often have cooccurring chronic illnesses, putting them at risk for higher healthcare utilization. ${ }^{8}$ Further, individuals with ADRD are not always able to advocate for themselves, making them potentially vulnerable to abuse. ${ }^{9}$ Given the current size of the AL industry and the large population of residents with ADRD, more information is needed about the care provided to individuals with dementia residing in AL. 
The development and growth of the AL industry has occurred largely without the influence of federal regulation. The lack of federal regulation is mainly a result of the funding structure of AL: in 2016, 84\% of residents paid privately for their care. ${ }^{1}$ With minimal federal oversight, state regulations dictate requirements for licensure and monitoring of AL. Previous work suggests that states vary widely in their regulations specific to the care of AL residents with ADRD ${ }^{10-14}$ and their enforcement practices. ${ }^{15}$ Regulation has also increased and diversified over time: in 2000, 28 states had at least one regulatory requirement for providing care to residents with ADRD in $\mathrm{AL},{ }^{16}$ and by 2014, that number had increased to 49 states. ${ }^{10}$ Given the differences across states in what is considered $\mathrm{AL}$ and the regulations related to caring for residents with ADRD (eg, staffing requirements, level of care permitted), it is important to examine state variation in the presence of $\mathrm{AL}$ residents with ADRD and their care experiences because regulations likely shape providers' behaviors and ultimately residents' outcomes.

The objectives of this study are to describe the state variability in the prevalence of ADRD among AL residents and their healthcare utilization. We use national Medicare claims data to characterize the sociodemographic and health characteristics of a cohort of Medicare beneficiaries with ADRD who reside in larger (25+ bed) AL settings and compare them with Medicare beneficiaries with ADRD living in the community and nursing homes (NHs). In addition, we present cross-sectional state variability in the prevalence of ADRD among a cohort of AL residents and their rates of $\mathrm{NH}$ admission and acute care hospitalization, and compare these rates with the population of communitydwelling Medicare beneficiaries with ADRD.

\section{METHODS}

\section{Data}

Information on $\mathrm{AL}$ communities comes from a national census we compiled from individual state licensing agencies. We reviewed state websites and contacted state agents for information on licensed $\mathrm{AL} /$ residential care settings in each state. Licensing agencies provided information, at a minimum, on the license type, address, and capacity. Following past work, we only include $\mathrm{AL}$ residences licensed to serve a population of older adults and with capacities for $25+$ residents. ${ }^{1,17-20}$ Although larger AL communities $(25+$ beds $)$ made up approximately $39.2 \%$ of AL communities in 2016, they comprised $84.1 \%$ of all licensed beds nationally. ${ }^{1}$ Therefore, residential care settings with fewer than 25 beds, which represent more than $60 \%$ of licensed care settings but only $15.9 \%$ of residents, were not included in our analyses.

The Medicare Master Beneficiary Summary File (MBSF) was used to obtain beneficiaries' age, race, sex, reason for Medicare entitlement, Medicaid eligibility, and date of death. The Chronic Conditions Warehouse (CCW) segment of the MBSF was used to identify beneficiaries' chronic conditions including ADRD (see Supplementary Material for additional details). The MBSF was linked to a ZIP Code History File to obtain beneficiaries' residential ZIP Codes.

To identify Medicare beneficiaries in AL, we updated a previously published methodology relying on the 9-digit
ZIP Code reported in the MBSF ${ }^{1}$ (see Supplemental Material for additional details) and created a finder file of 52,493 validated 9-digit ZIP Codes associated with 11,916 ALs (many large AL campuses have more than one 9-digit ZIP Code). Using this finder file, we searched beneficiaries' residential ZIP Codes to identify Medicare beneficiaries residing in large AL settings with a validated 9-digit ZIP Code pertaining to an AL on December 31, 2016.

To identify our cohorts of community-dwelling and $\mathrm{NH}$ residents, we combined Medicare claims with the Minimum Data Set and Home Health Outcome and Assessment Information Set assessments to form a Residential History File (RHF) that identifies a person's location of care and healthcare utilization on each day within a calendar year. ${ }^{21}$

\section{Sample}

We identified 439,272 Medicare beneficiaries with a ZIP Code pertaining to an $\mathrm{AL}$ in the 48 contiguous states who were not in any other healthcare setting on December 31, 2016. Because utilization and claims data are not available for Medicare Advantage (MA) beneficiaries, we excluded AL residents with any MA coverage during calendar years 2016 and 2017, resulting in 293,336 beneficiaries enrolled in traditional Medicare residing in AL on December 31, 2016. We used the CCW segment of the MBSF to identify residents with ADRD. This resulted in a sample of 88,867 (30.3\%) beneficiaries enrolled in traditional Medicare with an ADRD diagnosis residing in AL on December 31, 2016.

To compare our sample of AL residents with ADRD with other populations, we identified the population of Medicare beneficiaries with an ADRD diagnosis residing in the community (ie, were not in AL, an inpatient setting, or an NH) on December 31, 2016, and without any MA enrollment during 2016 and $2017(\mathrm{n}=2,074,420)$. We also identified the population of beneficiaries enrolled in traditional Medicare in 2016 and 2017 with an ADRD diagnosis who were in an NH on December 31, $2016(\mathrm{n}=602,521)$.

\section{Measures}

The RHF was used to create the following outcomes for each of the study cohorts: any NH admission (both post-acute and long-stay) and any inpatient acute hospitalization during 2017. Inpatient acute hospitalization was specified for $\mathrm{NH}$ residents as an inpatient admission while an $\mathrm{NH}$ resident. For $\mathrm{AL}$ and community residents, inpatient admissions were included if they occurred while in AL or the community (ie, hospitalizations from an $\mathrm{NH}$ were not included).

\section{Analyses}

We conducted descriptive analyses documenting the differences in demographic and health characteristics among Medicare beneficiaries with an ADRD diagnosis who resided in AL with those residing in the community or an $\mathrm{NH}$. We then calculated the percentage of AL residents with ADRD in each of the 48 states. Next, we estimated separate logistic regression models for each outcome measure (ie, $\mathrm{NH}$ admission and hospitalization) for $\mathrm{AL}$ and community-dwelling residents with ADRD. The models adjusted for age group $(\leq 64,65-74,75-84,85-94$, and $\geq 95$ 
y), sex, race, ${ }^{22}$ dual eligibility (ie, Medicare and Medicaid) status in December 2016, and the presence and number $(<2,2-3,4-5$, and $\geq 6)$ of the following chronic conditions: anemia, atrial fibrillation, cancer, chronic kidney disease, chronic obstructive pulmonary disease, depression, diabetes, heart failure, hyperlipidemia, hypertension, ischemic heart disease, and stroke.

We calculated the risk-adjusted rates of each outcome, by state, as the ratio of observed events over expected events in each state, multiplied by the national rate. The risk-adjusted data were plotted to compare the rates of the outcomes in AL vs the community. The bootstrap resampling method was performed to estimate the $95 \%$ confidence intervals (CIs) of adjusted rates, and two-sample $t$ tests were used to compare adjusted group means. All analyses were approved by the Brown University institutional review board and conducted with SAS v.9.4 (SAS Institute, Cary, NC) and Stata v.15.1 (StataCorp 2017, College Station, TX). Additional information about the data and methods used for these analyses can be found in the Brown University Digital Repository (https://repository.library. brown.edu/studio/item/bdr:1078482/).

\section{RESULTS}

A total of $88,867(30.3 \%)$ beneficiaries enrolled in traditional Medicare residing in AL had a diagnosis of ADRD, compared with $6.1 \%$ in the community and $72.7 \%$ in NHs (Table 1 ).

Table 1. Demographic and Health Characteristics of Assisted Living Residents with a Diagnosis of Alzheimer's Disease and Related Dementias Compared with Community-Dwelling and Nursing Home Residents

\begin{tabular}{|c|c|c|c|}
\hline & $\begin{array}{c}\text { Assisted living } \\
\mathrm{N}=\mathbf{8 8 , 8 6 7}(\mathbf{3 0 . 3} \%)\end{array}$ & $\begin{array}{c}\text { Community } \\
N=2,074,420(6.1 \%)\end{array}$ & $\begin{array}{c}\text { Nursing home } \\
\mathrm{N}=602,521(72.7 \%)\end{array}$ \\
\hline \multicolumn{4}{|l|}{ Age groups, ${ }^{a} y, n(\%)$} \\
\hline $65-74$ & 7,587 (8.5) & $362,158(17.5)$ & $83,208(13.8)$ \\
\hline $75-84$ & $19,604(22.1)$ & $727,822(35.1)$ & $169,192(28.1)$ \\
\hline $85-94$ & $45,869(51.6)$ & $726,491(35)$ & $243,909(40.5)$ \\
\hline \multicolumn{4}{|l|}{ Sex, $n(\%)$} \\
\hline Male & $27,107(30.5)$ & $797,248(38.4)$ & $187,800(31.2)$ \\
\hline Female & $61,760(69.5)$ & $1,277,171(61.6)$ & $414,721(68.8)$ \\
\hline \multicolumn{4}{|l|}{ Race, n (\%) } \\
\hline White & 81,624 (91.9) & $1,642,568(79.2)$ & $478,472(79.4)$ \\
\hline Black & $3,668(4.1)$ & $199,346(9.6)$ & $74,269(12.3)$ \\
\hline Anemia & $39,457(44.4)$ & $791,298(38.2)$ & $342,002(56.8)$ \\
\hline Atrial fibrillation & $16,474(18.5)$ & $300,995(14.5)$ & $106,911(17.7)$ \\
\hline Cancer & $8,833(9.9)$ & $205,119(9.9)$ & $47,194(7.8)$ \\
\hline Chronic kidney disease & $34,101(38.4)$ & $774,811(37.4)$ & $274,375(45.5)$ \\
\hline Chronic obstructive pulmonary disease & $17,909(20.2)$ & $384,770(18.6)$ & $149,652(24.8)$ \\
\hline Depression & $38,697(43.5)$ & $698,182(33.7)$ & $339,951(56.4)$ \\
\hline Diabetes & $27,588(31.0)$ & $733,149(35.3)$ & $256,216(42.5)$ \\
\hline Heart failure & 29,525 (33.2) & $569,210(27.4)$ & $250,358(41.6)$ \\
\hline Hyperlipidemia & $46,799(52.7)$ & $1,134,061(54.7)$ & $295,509(49.1)$ \\
\hline Hypertension & $71,137(80.1)$ & $1,555,130(75)$ & $508,506(84.4)$ \\
\hline Ischemic heart disease & $40,351(45.4)$ & $897,237(43.3)$ & $289,819(48.1)$ \\
\hline No. of hospitalizations, ${ }^{d}$ mean (standard deviation) & $1.48(.92)$ & $1.58(1.11)$ & $1.55(1.02)$ \\
\hline Mortality rate, ${ }^{\mathrm{b}} \mathrm{n}(\%)$ & $17,491(19.7)$ & $303,891(14.7)$ & $187,350(31.1)$ \\
\hline
\end{tabular}

${ }^{\mathrm{a}}$ As of December 31, 2016.

${ }^{\mathrm{b}}$ During 2017.

cNursing home admission and/or hospitalization occurred while in that setting.

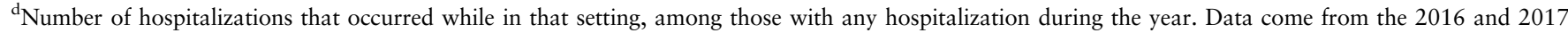

Medicare Master Beneficiary Summary File, chronic conditions, and Residential History File. 


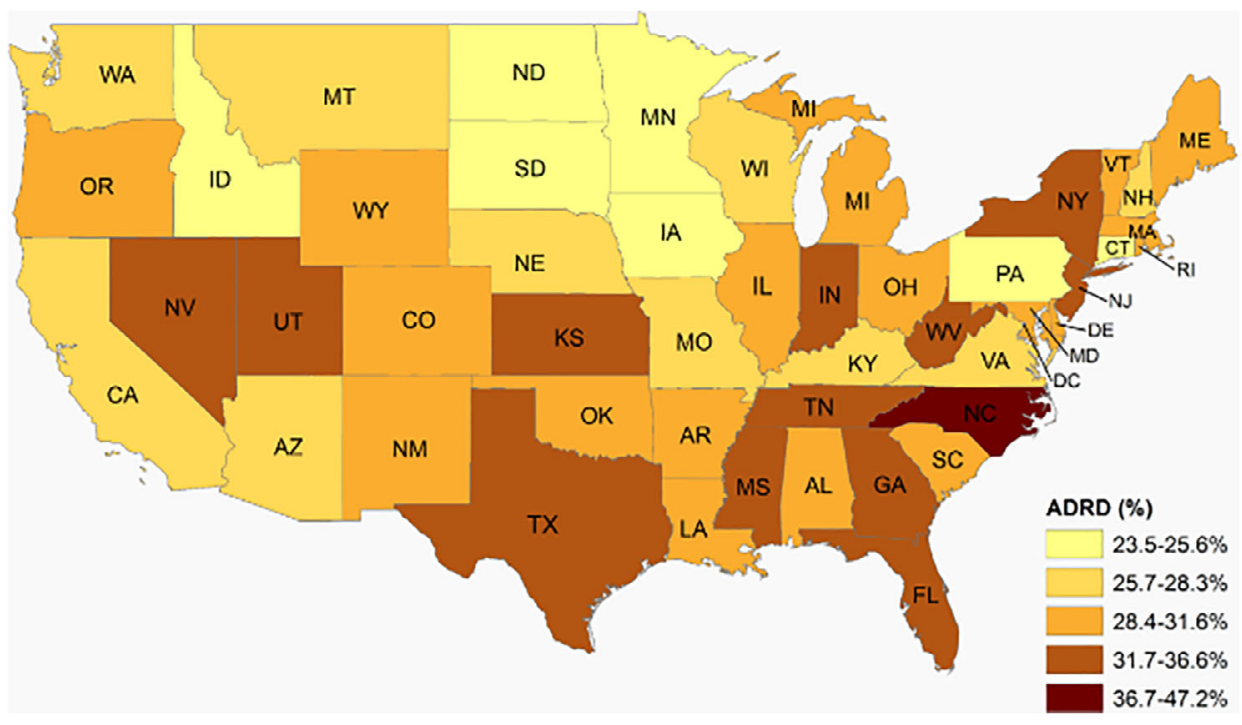

Figure 1. Geographic variability in the share of beneficiaries enrolled in traditional Medicare residing in assisted living with a diagnosis of Alzheimer's disease and related dementias (ADRD) (2016). Data come from the 2016 Medicare Master Beneficiary Summary File and chronic conditions segment.

Our national cohort of beneficiaries with ADRD residing in $\mathrm{AL}$ were more likely to be older, female, and white than Medicare beneficiaries with a diagnosis of ADRD living in the community or $\mathrm{NH}$. AL residents had more diagnosed chronic conditions than the community-dwelling population with ADRD but fewer than NH residents with ADRD. In addition, AL residents with ADRD had rates of hospitalization higher than community-dwelling and $\mathrm{NH}$ residents with $\operatorname{ADRD}(37.6 \%$ vs $33.6 \%$ and $28.9 \%$, respectively).

Among our cohort of AL residents, states varied in their unadjusted percentage of residents with an ADRD diagnosis, from $23.6 \%$ in Minnesota to $47.2 \%$ in North Carolina (Figure 1 and Supplementary Table S1). In 2017, a total of
21,151 (23.8\%) AL residents with an ADRD diagnosis were admitted to an NH during the year compared with $16.5 \%$ of community-dwelling beneficiaries with ADRD. The adjusted percentage of AL residents with an $\mathrm{NH}$ admission varied by state, ranging from $13.7 \%(95 \% \mathrm{CI}=9.7-18.7)$ in New Mexico to $34.9 \%(95 \% \mathrm{CI}=29-40.5)$ in North Dakota. Figure 2 shows that the rate of $\mathrm{NH}$ admission among AL residents is higher than in the community, but that state-level rates are strongly correlated $(r=.74)$. With few exceptions, states whose community rates of $\mathrm{NH}$ admission were above/below the median also had higher/lower $\mathrm{NH}$ admission among AL residents. Delaware, North Dakota, and Connecticut had the greatest absolute difference in the rate of $\mathrm{NH}$ admission

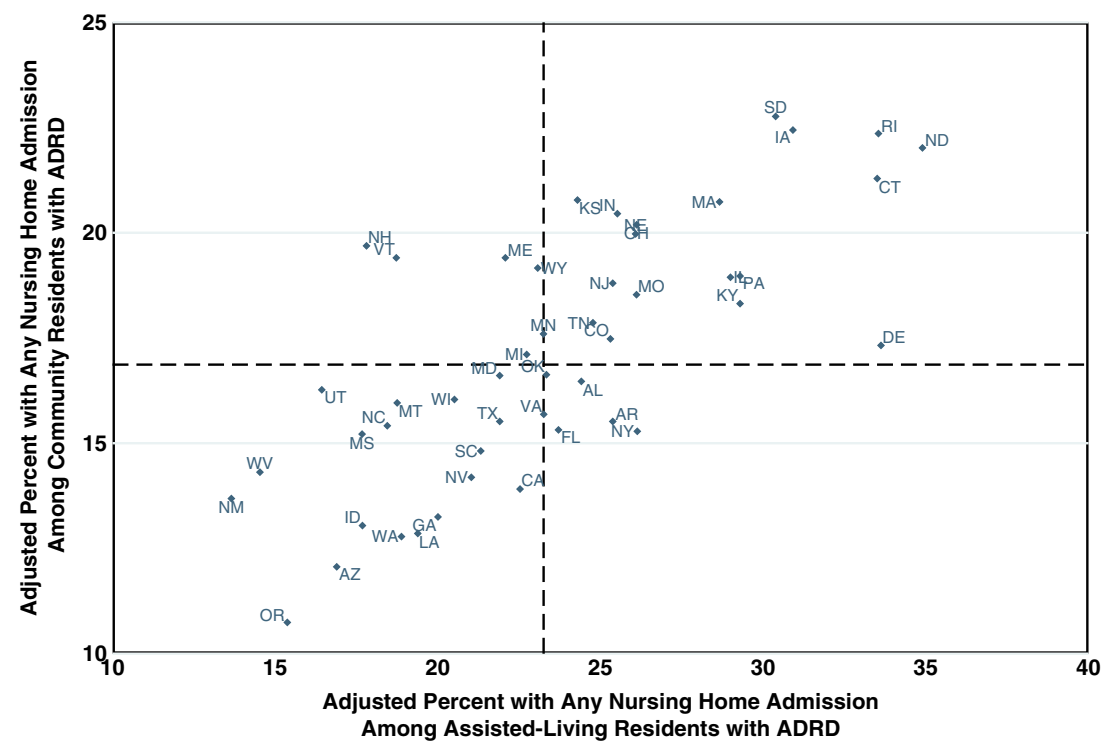

Figure 2. Adjusted* percentage of Medicare beneficiaries with Alzheimer's disease and related dementias (ADRD) with a nursing home admission in 2017. *Percentage adjusted for age, race, sex, dual eligibility, chronic conditions, and the number of chronic conditions. Data come from the 2016 and 2017 Medicare Master Beneficiary Summary File and the Residential History File. Dashed lines indicate median values. [Color figure can be viewed at wileyonlinelibrary.com] 


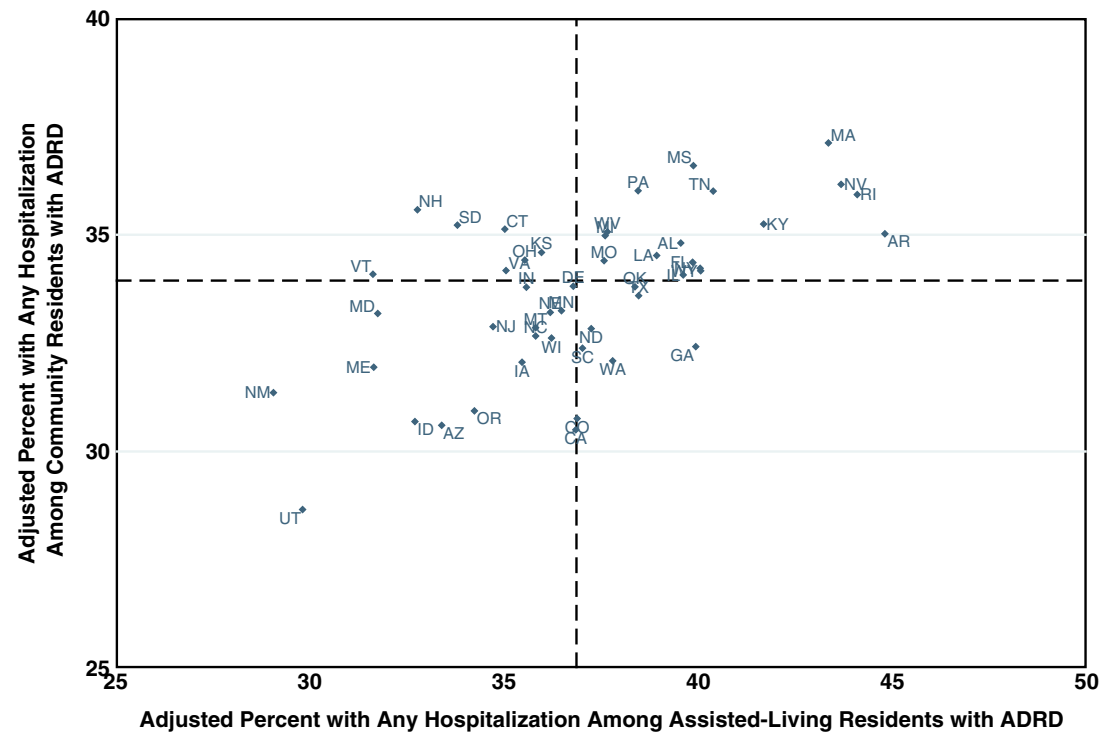

Figure 3. Adjusted* percentage of Medicare beneficiaries with Alzheimer's disease and related dementias (ADRD) with a hospitalization in 2017. *Percentage adjusted for age, race, sex, dual eligibility, chronic conditions, and the number of chronic conditions. Hospitalizations occurred before moving to a nursing home. Data come from the 2016 and 2017 Medicare Master Beneficiary Summary File and the Residential History File. Dashed lines indicate median values. [Color figure can be viewed at wileyonlinelibrary.com]

between AL and community-dwelling beneficiaries with ADRD, with a more than 12 percentage point higher rate in $\mathrm{AL}$ than the community (Figure 2 and Supplementary Table S2). The NH admission rate in the community was only nominally higher but not significantly different from AL $(<2$ percentage point difference) in two states: New Hampshire and Vermont.

In our cohort, 33,457 (37.6\%) AL residents with ADRD were hospitalized during the year compared with $33.6 \%$ of community-dwelling beneficiaries with ADRD. The adjusted percentage of AL residents with ADRD hospitalized within the year varied by state and ranged from $29.1 \%(95 \% \mathrm{CI}=$ 23.4-35.9) in New Mexico to $44.8 \%$ (95\% CI $=40.7-49.2)$ in Arkansas (Figure 3). When comparing the adjusted rates of hospitalization in AL vs the community, Arkansas, Rhode Island, Georgia, and Nevada had the greatest absolute difference ( $>7$ percentage points) in the adjusted rate of hospitalization (Supplementary Table S3). The rate of hospitalization in the community was nominally higher $(<3$ percentage points) but not significantly different from AL in seven states: New Hampshire, Vermont, New Mexico, Maryland, South Dakota, Maine, and Connecticut.

\section{DISCUSSION}

This analysis is the first to compare the sociodemographic and health characteristics of Medicare beneficiaries with ADRD who reside in AL with those living in the community and NHs. We found that several measures of health of our national cohort of AL residents with ADRD fell in between ADRD patients in the community and those in NHs. Among $\mathrm{AL}$ residents, we observed state variation in the presence of ADRD, suggesting potential differences in access to $\mathrm{AL}$ for patients with ADRD. This article is also the first to examine the healthcare utilization of AL residents with ADRD by state. We found that the rates of $\mathrm{NH}$ admission and hospitalization among our cohort of AL residents varied dramatically across the country, and, on average, they were higher than community-dwelling beneficiaries with ADRD. In the following paragraphs, we describe some of the potential mechanisms behind the state variability we observed in this study.

Our findings suggest that states vary in the proportion of $\mathrm{AL}$ residents with an ADRD diagnosis. This interstate variability could be a function of market forces, regulatory requirements, and AL provider behavior. For example, it may be that there are geographic differences in the clinical diagnosis of ADRD, as was previously observed across states and rural/urban locations. ${ }^{23,24}$ The variation in the prevalence of ADRD in AL could also be a function of differences in states' long-term services and supports available to care for individuals with ADRD. ${ }^{25}$

In addition to availability, states may vary in how they assist residents in financing AL. Given that close to onequarter of all patients with ADRD in the community are enrolled in Medicaid, ${ }^{26}$ the availability of public subsidies (eg, Medicaid benefits for services provided in AL and generosity of state supplements to pay for care in $\mathrm{AL}^{10,27}$ ) also may be driving the state variability in $\mathrm{AL}$ residents with ADRD. Notably, Pennsylvania, a state with one of the lowest prevalence rates of ADRD among AL residents (24.9\%), despite being home to one of the oldest populations in the country, ${ }^{28}$ is one of a handful that does not provide services funded by Medicaid to residents in AL.

The variation in states' regulations pertaining to dementia care may also be associated with variation in the prevalence of ADRD in AL across states. For example, some states require dementia-specific preadmission screening (14 states), consumer disclosure (33 states), and building design to accommodate the needs of residents with ADRD (29 states), ${ }^{11}$ and these differences likely impact AL providers' willingness to accept residents with an ADRD diagnosis. Future research is needed to better understand the market and regulatory factors that may contribute to the state variability in the prevalence of ADRD among AL residents. 
We also observed significant state variation in the rates of using $\mathrm{NH}$ care among AL residents with ADRD. Two types of factors may contribute to variation in $\mathrm{NH}$ admission rates: those that affect both community and AL residents, and those specific to AL. The mechanisms driving the differences in $\mathrm{NH}$ admission in both settings, therefore leading to strong correlation between community and AL rates, are likely related to potential factors contributing to differential access, as listed earlier (eg, market factors). For example, the number of $\mathrm{NH}$ beds per capita vary across the country, as does post-acute skilled nursing facility utilization. ${ }^{29,30}$ It may be the case that in states with less robust Medicaid support for home- and community-based services, individuals with ADRD living in the community may be left with few care alternatives to NHs.

The variation in $\mathrm{NH}$ placement among $\mathrm{AL}$ residents across states may also reflect differences in Medicaid policies: states without Medicaid waivers or state plans allowing for services to be provided in AL may result in residents spending down their assets, requiring relocation to an $\mathrm{NH}$ or other setting that accepts Medicaid reimbursement. State regulations related to retention or move-out requirements may affect the case mix within $\mathrm{AL},{ }^{31}$ thereby potentially driving the differences in $\mathrm{NH}$ admission across states.

In North Dakota, the state with the highest $\mathrm{NH}$ admission rate, $\mathrm{AL}$ settings may not retain residents who require more than intermittent nursing care unless the resident requires and elects to receive end-of-life care from a Medicare-certified hospice agency and the AL is licensed to provide end-of-life care (NDAC 33-03-24.1). ${ }^{32}$ This policy might result in transfers of residents with ADRD who have nursing care needs that are not deemed as life limiting.

State-specific dementia care rules might also influence the number of individuals with ADRD who are able to maintain residence in $\mathrm{AL}$ as their disease progresses. For example, Vermont, a state with a higher $\mathrm{NH}$ admission rate in the community than AL, has a policy that residents "will be permitted to age in place" (CVR 13-110-007.6.5) ${ }^{33}$ provided that their mobility, ambulation, and transfer needs can be met by one staff person, cognitive impairment is at a moderate or lesser degree of severity, and behavioral symptoms consistently respond to appropriate intervention.

Other regulatory requirements, such as staffing, admission criteria, care processes, and tolerance for aging in place, might also explain the differences in $\mathrm{NH}$ admission observed among states. Our findings set the stage for future work examining the relative contribution of these market, financing, and regulatory factors on the state variations observed in this study.

Nationally, our cohort of AL residents with ADRD had higher rates of hospitalization than communitydwelling and $\mathrm{NH}$ residents, and the differences between $\mathrm{AL}$ and community rates varied by state. These rates might reflect differences in quality of care provided in $\mathrm{AL}$, state requirements for level of care to be provided, or underlying patient need. It is impossible to draw conclusions about the quality of care provided in AL because systematic unmeasured aspects of health, such as the level of functional and cognitive impairment, could contribute to AL residents' differences in hospitalization rates. Additional work examining hospitalizations conditioned on underlying patient need is important and required to better understand the quality of care provided to residents with $\mathrm{ADRD}$ in $\mathrm{AL}$ compared with the community and NHs.

As it relates to state regulations for dementia care requirements, of the four states with the greatest differences in $\mathrm{AL}$ and community rates of hospitalization, three (Arkansas, Rhode Island, and Nevada) require dementia care units to be licensed. However, of the seven states with lower rates of hospitalization in $\mathrm{AL}$, only Connecticut requires dementia care units to be licensed. ${ }^{11}$ It is possible that states with more stringent rules for dementia care settings have provisions that result in differential rates of hospitalization or that they admit residents with differing levels of need. However, documentation of state variation in dementia care requirements for AL is limited.

One regulatory review found that only seven states specify minimum staffing levels or ratios in dementia care units; 13 states specified administrator training requirements, with the total hours of training ranging from 7 to 120 hours in the first year, and 14 states required a preadmission assessment of dementia care needs. ${ }^{11}$ Given the previous link between nurse staffing levels, staffing mix, and AL residents' outcomes, ${ }^{34,35}$ it is possible that state staffing requirements may also be associated with differences in rates of hospitalization. Future research is needed to assess systematically whether and how differences in states' requirements for dementia care, particularly staffing levels and mix, affect hospitalization of AL residents with ADRD.

Differences among states in healthcare utilization of their AL residents may be a function not only of states' regulations but also how those regulations are monitored and enforced. Although the relationship between enforcement of regulations and resident outcomes has not been explored in the context of $\mathrm{AL}$, the link between oversight and quality of care in NHs has been well documented. For example, implementation of the Nursing Home Reform Act care standards and surveillance system was tied to reductions in chemical restraints and improved resident quality of care. ${ }^{36}$ Following the nationwide implementation of the National Partnership to Improve Dementia Care in Nursing Homes, one study ${ }^{37}$ found state agencies supporting frequent facility monitoring had reduced rates of inappropriate antipsychotic prescribing. Additional work to understand the relationship between how states enforce regulations and residents' outcomes is an area of important study.

\section{Limitations}

This analysis has limitations. First, we present a cross section of data and report on the resident composition and healthcare utilization in 2017; therefore, we are unable to draw any causal conclusions. As such, we do not formally measure the impact of market factors, regulations, or enforcement that may be attributable to the geographic variation observed.

Second, our ascertainment of ADRD relies on a diagnosis in Medicare claims. Therefore, we may be underestimating the prevalence of residents with $\mathrm{ADRD}^{4}$ and are unable to identify the prevalence of ADRD among MA enrollees. Given the varying diagnosing patterns ${ }^{23,24}$ and MA penetration rate across states, ${ }^{38}$ this may bias our estimates of ADRD prevalence in these settings. However, it does not appear that AL prevalence rates across 
states track those among community-dwelling older adults (Supplementary Table S1).

Third, our methodology depends on identifying residents in larger AL settings (25+ beds), limiting its generalizability to smaller settings. Data from the National Study of Long-Term Care Providers (NSLTCP) suggests that the percentage of residents with ADRD decreases with increasing $\mathrm{AL}$ size, from $51 \%$ of residents in $\mathrm{AL}$ settings with 4 to 25 beds, to $44 \%$ with 26 to 50 beds, to $39 \%$ in the largest AL settings (50+ beds). Understandably, our study had lower rates of ADRD than previously published findings from the 2016 NSLTCP. ${ }^{39}$ In addition, relying on 9-digit ZIP Codes to identify AL residents means that we are only able to identify residents who changed their addresses, and we may be including independent living residents on the same campus.

Finally, this study was designed and carried out using the state as the primary unit of analysis. However, most states provide two or more types of licensed care that falls under the umbrella of $\mathrm{AL}$, and requirements for licensed settings within a state can vary. Additional investigation is needed to better understand the within-state variability that is likely to exist in resident characteristics and outcomes. Despite these limitations, our study presents the first insight into the state variability in the prevalence of ADRD and residents' healthcare utilization.

In conclusion, for several decades, AL has been preferred by older adults who need assistance with personal care and health-related needs. As this and other recent studies have indicated, ${ }^{1-7}$ AL serves significant numbers of older adults with ADRD. The state variation in the percentage of residents with ADRD diagnoses, and the variation in their health service utilization, suggest unequal access to AL, differences in care processes, as well as possible underlying variability in the population of beneficiaries with ADRD residing in AL. The reasons for such differences are as yet unknown, although market forces, regulatory requirements, and enforcement may play important roles. Our findings suggest that clinicians caring for residents in these settings likely practice under varying constraints. These findings also point to the importance of clinicians' familiarity with state and specific AL communities' requirements for care. Ultimately, these findings call for further work to better understand the factors that might be driving the variations observed in this study.

\section{ACKNOWLEDGMENTS}

Financial Disclosure: This study was supported by the National Institute of Aging (R01 AG057746; PI: KST) and the US Department of Veterans Affairs Health Services Research and Development Service (CDA 14-422; PI: KST).

Conflict of Interest: The authors have declared no conflicts of interest for this article.

Author Contributions: Study concept and design: Thomas and Cornell. Acquisition of data: Thomas. Analysis and interpretation of data: Thomas, Zhang, and Cornell. Drafting of the manuscript: All authors. Critical revision of the manuscript for important intellectual content: All authors.
Sponsor's Role: The sponsors had no role in the design, methods, subject recruitment, data collections, analysis, or preparation of the article.

\section{REFERENCES}

1. Harris-Kojetin L, Sengupta M, Lendon JP, Rome V, Valverde R, Caffrey C. Longterm care providers and services users in the United States, 2015-2016. National Center for Health Statistics. Vital Health Stat. 2019;3(43). https://www.cdc.gov/ nchs/data/series/sr_03/sr03_43-508.pdf. Accessed October 9, 2019.

2. Thomas KS, Dosa D, Gozalo PL, et al. A methodology to identify a cohort of Medicare beneficiaries residing in large assisted living facilities using administrative data. Med Care. 2018;56(2):e10-e15.

3. Zimmerman S, Sloane PD, Reed D. Dementia prevalence and care in assisted living. Health Aff (Millwood). 2014. 33(4):658-666.

4. Magsi H, Malloy T. Under-recognition of cognitive impairment in assisted living facilities. J Am Geriatr Soc. 2005;53(2):295-298.

5. Caffrey C, Sengupta M, Park-Lee E, Moss A, Rosenhoff E, Harris-Kojetin L. Residents living in residential care facilities: United States, 2010. NCHS Data Brief. 2012;(91):1-8.

6. Golant SM. Do impaired older persons with health care needs occupy U.S. assisted living facilities? An analysis of six national studies. J Gerontol B Psychol Sci Soc Sci. 2004;59(2):S68-S79.

7. Rosenblatt A, Samus QM, Steele CD, et al. The Maryland Assisted Living Study: prevalence, recognition, and treatment of dementia and other psychiatric disorders in the assisted living population of central Maryland. J Am Geriatr Soc. 2004;52(10):1618-1625.

8. Alzheimer's Association. 2019 Alzheimer's disease facts and figures. Alzheimers Dement. 2019;15(3):321-387. https://www.alz.org/media/documents/alzheimersfacts-and-figures-2019-r.pdf. Accessed January 27, 2020.

9. Dong X, Chen R, Simon MA. Elder abuse and dementia: a review of the research and health policy. Health Aff. 2014;33(4):642-649.

10. Carder PC, O'Keeffe J, O'Keeffe C. Compendium of Residential Care and Assisted Living Regulations and Policy: 2015 Edition Executive Summary. https:// aspe.hhs.gov/basic-report/compendium-residential-care-and-assisted-livingregulations-and-policy-2015-edition. Accessed October 9, 2019.

11. Carder PC. State regulatory approaches for dementia care in residential care and assisted living. Gerontologist. 2017;57(4):776-786.

12. Nattinger MC, Kaskie B. Determinants of the rigor of state protection policies for persons with dementia in assisted living. J Aging Soc Policy. 2017;29 (3):123-142.

13. Kaskie BP, Nattinger M, Potter A. Policies to protect persons with dementia in assisted living: deja vu all over again? Gerontologist. 2015;55(2):199-209.

14. Kaskie B, Knight BG, Liebig PS. Dementia policy revisited: an analysis of the state laws targeted towards individuals with Alzheimer's disease and other forms of dementia. J Aging Soc Policy. 2001;12(4):27-45.

15. Kaskie B, Kingsley K. State Enforcement Actions Pertaining to Persons with Dementia Residing in Assisted Living Facilities. Washington, DC: Alzheimer's Association National Public Policy Office; 2009.

16. Mollica R, Sims-Kastelein K, O'Keefe J. Residential Care and Assisted Living Compendium. Washington, DC: US Department of Health and Human Services; 2007. https://aspehhsgov/system/files/pdf/75316/07alcom.pdf. Accessed October 9, 2019.

17. Stevenson DG, Grabowski DC. Sizing up the market for assisted living. Health Aff (Millwood). 2010;29(1):35-43.

18. Grabowski DC, Stevenson DG, Cornell PY. Assisted living expansion and the market for nursing home care. Health Serv Res. 2012;47(6):2296-2315.

19. Silver BC, Grabowski DC, Gozalo PL, Dosa D, Thomas KS. Increasing prevalence of assisted living as a substitute for private-pay long-term nursing care. Health Serv Res. 2018;53(6):4906-4920.

20. Thomas KS, Belanger E, Zhang W, Carder P. State variability in assisted living residents' end-of-life care trajectories. J Am Med Dir Assoc. 2020;21(3):415419. https://doi.org/10.1016/j.jamda.2019.09.013.

21. Intrator O, Hiris J, Berg K, Miller SC, Mor V. The residential history file: studying nursing home residents' long-term care histories. Health Serv Res. 2011;46(1 Pt 1):120-137.

22. Eicheldinger C, Bonito A. More accurate racial and ethnic codes for Medicare administrative data. Health Care Financ Rev. 2008;29(3):27-42.

23. Russ TC, Batty GD, Hearnshaw GF, Fenton C, Starr JM. Geographical variation in dementia: systematic review with meta-analysis. Int J Epidemiol. 2012;41(4):1012-1032.

24. Koller D, Bynum JPW. Dementia in the USA: state variation in prevalence. J Public Health (Oxf). 2015;37(4):597-604.

25. Houser A, Fox-Grage W, Ujvari K. Across the States: Profiles of Long-Term Services and Supports. AARP Public Policy Institute; 2018. https://www.aarp.org/ 
content/dam/aarp/ppi/2018/08/across-the-states-profiles-of-long-term-servicesand-supports-full-report.pdf. Accessed October 9, 2019.

26. Garfield R, Musumeci MB, Reaves E, Damico A. Medicaid's Role for People with Dementia. Kaiser Family Foundation; 2015. https://www.kff.org/medicaid/ issue-brief/medicaids-role-for-people-with-dementia/. Accessed October 9, 2019.

27. Department of Health and Human Services Report. Home and CommunityBased Services in Assisted Living Facilities; 2012. https://oig.hhs.gov/oei/ reports/oei-09-08-00360.pdf. Accessed October 9, 2019.

28. Himes CL. Which U.S. States Are the Oldest? Population Reference Bureau; 2003. https://www.prb.org/whichusstatesaretheoldest/. Accessed October 18, 2019.

29. Wang Y, Zhang Q, Spatz ES, et al. Persistent geographic variations in availability and quality of nursing home care in the United States: 1996 to 2016. BMC Geriatr. 2019;19:103.

30. Newhouse JP, Garber A, Graham RP, eds. Interim Report of the Committee on Geographic Variation in Health Care Spending and Promotion of High-Value Health Care: Preliminary Committee Observations. Washington, DC: National Academies Press; 2013. https://www.ncbi.nlm.nih.gov/books/NBK201532/. Accessed October 29, 2019.

31. Kissam S, Gifford DR, Mor V, Patry G. Admission and continued-stay criteria for assisted living facilities. J Am Geriatr Soc. 2003;51:1651-1654.

32. North Dakota Administrative Code-Chapter 33-23-24.1. Eff 2018. https:// www.legis.nd.gov/information/acdata/pdf/33-03-24.1.pdf. Accessed January 27, 2020.

33. Vermont Administrative Code-Chapter 13-110-007. Eff 2013. https://dail. vermont.gov/sites/dail/files//documents/Assisted_living_regs_final_2003.pdf. Accessed January 29, 2020.

34. Zimmerman S, Sloane PD, Eckert JK, et al. How good is assisted living? Findings and implications from an outcomes study. J Gerontol B Psychol Sci Soc Sci. 2005;60(4):S195-S204.

35. Stearns SC, Park J, Zimmerman S, Gruber-Baldini AL, Konrad TR, Sloane PD. Determinants and effects of nurse staffing intensity and skill mix in residential care/assisted living settings. Gerontologist. 2007;47(5):662-671.

36. Fashaw SA, Thomas KS, McCreedy E, Mor V. Thirty-year trends in nursing home composition and quality since the passage of the Omnibus Reconciliation Act. J Am Med Dir Assoc. 2019;21(2):233-239.
37. Urick BY, Kaskie BP, Carnahan RM. Improving antipsychotic prescribing practices in nursing facilities: the role of surveyor methods and surveying agencies in upholding the nursing home reform act. Res Social Adm Pharm. 2016;12(1):91-103.

38. Jacobson G, Freed M, Damico A, Neuman T. A Dozen Facts About Medicare Advantage in 2019. Kaiser Family Foundation; 2019. https://www.kff. org/medicare/issue-brief/a-dozen-facts-about-medicare-advantage-in-2019/. Accessed October 29, 2019.

39. Lendon JP, Sengupta M, Rome V, Caffrey C, Harris-Kojetin L, Melekin A. Long-Term Care Providers and Services Users in the United States-State Estimates Supplement: National Study of Long-Term Care Providers, 2015-2016. National Center for Health Statistics: Hyattsville, MD; 2019 https://www.cdc.gov/nchs/data/nsltcp/2016_CombinedNSLTCPStateTables_ opt.pdf. Accessed November 12, 2019.

\section{SUPPORTING INFORMATION}

Additional Supporting Information may be found in the online version of this article.

Supplementary Table S1: Unadjusted rate of Alzheimer's disease and related dementias (ADRD) by state.

Supplementary Table S2: Adjusted rates of moving into a nursing home among residents with an Alzheimer's disease and related dementias (ADRD) diagnosis in assisted living and community.

Supplementary Table S3: Adjusted rates of hospitalization before moving to a nursing home among residents with an Alzheimer's disease and related dementias (ADRD) diagnosis in assisted living and community. 